\title{
La "formazione" della contingenza: Se una notte d'inverno e il "racconto" lacaniano sull'amore
}

Il capitolo sesto, centrale in Se una notte d'inverno un viaggiatore, presenta, in "memoria" della tradizione retorica, la classica "invocazione alla musa" e un'importante chiave di lettura. È il capitolo dedicato alle varie metamorfosi della "musa" Lettrice di cui la prima, "la Sultana che, in base al contratto matrimoniale, deve ricevere una congrua dose quotidiana di narrativa, è una reincarnazione di Sharazad . . ." (Segre 183). Ma la molteplicità delle immagini della Lettrice $^{1}$ ne costituiscono un trascendimento a favore della funzione stessa della lettura come matrice del desiderio da cui origina e verso cui tende la scrittura. In questo senso la Lettrice è la nuova Sharazad che mantiene in vita col suo bisogno mai appagato la narrazione; una narrazione "in crisi", quella di un autore che "secondo le voci più pessimistiche, si sarebbe messo a scrivere un diario, un quaderno di riflessioni, in cui non succede mai niente, solo i suoi stati d'animo e la descrizione del paesaggio che egli contempla per ore dal balcone, attraverso un cannocchiale..." (Se una notte 121).

È ovvio il riferimento a Palomar e a quel "blocco" di Calvino nei confronti del "racconto" di cui Se una notte rappresenta la soluzione. L" invocazione" al desiderio della lettura diviene la necessaria premessa all'inasprirsi delle difficoltà del "viaggio": qui la meditazione sui dubbi e le incertezze dello scrivibile. Inoltre, i capitoli sesto, settimo e ottavo possono essere letti come una rifrazione al livello metaforico del romanzo di quella tematica dell" "incontro" che, come vedremo, percorre sia i romanzi inseriti che la cornice. Proprio in questa direzione, c'è una storia (la decima ed ultima di quelle inserite nella comice) che merita particolare attenzione perché, riprendendo noti motivi calviniani, riscrive il finale del Bildungsroman tradizionale, ${ }^{2}$ qui, in "Quale storia laggiù attende la fine?" e, subito dopo, nella cornice, suggerendo come lettura parallela il "depotenziamento" del pensiero del "racconto" lacaniano sull'amore.

La storia è quella di un delirio di potenza, di controllo, di possibilità assoluta, di creatività sterminata. La città scompare sotto lo sguardo vittorioso del narratore che cancella burocrazie e gerarchie, regole e situazioni stabilite, con palazzi e uffici, impiegati e funzionari, passanti e sconosciuti: semplificare il mondo, eliminare impegni e costrizioni, ridurre all'essenziale, estrarre dal cumulo della fastidiosa rete di relazioni obbligate il nucleo di piacere, di libertà, di scelta, di "allegria". 
È un'esperienza già descritta in Marcovaldo in "La città smarrita nella neve": svegliarsi un mattino e trovare il mondo trasformato. Non più il regno del dovere ma del possibile, quello della creazione, della "scrittura": “. . . la città non c'era più, era stata sostituita da un foglio bianco" (Marcovaldo 25). Qualcosa è però cambiato: il personaggio di vent'anni-dopo-Marcovaldo sa "giocare" con le proprie potenzialità. Il possibile non è più il dono naturale, la "neve" fortuita che crea lo spazio del desiderio; chi parla (e scrive) costruisce orizzonti e sa di poterli distruggere: "Ora mi spiego la sensazione che avevo d'essere andato più in là d'altre volte nell'esercizio di far sparire il mondo che mi circonda" (Se una notte 251-52). Ma la dissoluzione del mondo, il mondo come nulla è pur sempre al di là dell'intenzione; è ancora l' "evento", il mistero, il "segreto" che si stende oltre la fragile linea che separa il "sempre" dal "mai", il tutto dal niente; è il "baratro", il "burrone", l'"abisso"; è, di nuovo, il "foglio di carta" ma su cui "non si riescono a scrivere altro che parole astratte, come se tutti i nomi concreti fossero finiti" (254).

Cesare Segre, a proposito di “Quale storia laggiù attende la fine?", osserva: "Un sogno, probabilmente, che si riferisce in modo abbastanza evidente ai problemi dell'espressione letteraria e, prima ancora, della nominazione" (207). Ma il problema qui posto da Calvino (come negli altri romanzi inseriti), non riguarda solo il rapporto mondo-rappresentazione o mondo-finzione ma (e soprattutto) il rapporto mondo-nulla. Il dramma del suo protagonista è il "vuoto": “. . . il vuoto è sempre più vuoto ... il mondo che io credevo cancellato da una decisione della mia mente che potevo rievocare in ogni momento, era finito davvero" (Se una notte 252). Siamo dunque in quell' area di "vertigini e oscuri presentimenti" che Segre considera marginali in questo testo (e forse in tutto Calvino) mentre, a mio parere, e come altri hanno già osservato, costituiscono una caratteristica essenziale del percorso calviniano. ${ }^{3}$

In "La città smarrita nella neve", per Marcovaldo il sogno fantasia-potenza, illusione-sovvertimento si spegne in un vorticar di tormenta che distrugge con la neve lo spazio mentale del possibile. La "natura", il caso, la circostanza, produrranno in avventure successive l'apertura di nuovi squarci, parentesi di "scrittura" di una quotidianità velocemente illuminata dall'illusione. Ma i suoi oscillamenti tra realtà e desiderio gli restano sconosciuti: la vita è sogno e il sogno è realtà. L'ambiguità fantastica è la via di fuga del personaggio. Per l'autore è già (o ancora) l'immagine di un'esperienza "indebolita". In Se una notte d'inverno, il "desiderio" e il "nulla" sono invece sulla pagina, sono ciò di cui si parla: sono i due opposti da coniugare, da far convivere nella loro irriducibile diversità.

Nel 1977, come "lettura montaliana" in occasione dell'ottantesimo compleanno del poeta, la scelta di Calvino (sono gli anni in cui sta scrivendo Se una notte d'inverno un viaggiatore), cade su "Forse un mattino andando in un'aria di vetro". Così il commento:

A ben vedere, la molla che scatena il "miracolo" è l'elemento naturale, cioè atmosferico, l'asciutta cristallina trasparenza dell'aria invernale, che rende le cose tanto 
nitide da creare un effetto d'irrealtà, quasi che l'alone di foschia che abitualmente sfuma il paesaggio ... s'identifichi con lo spessore e peso dell'esistere . . . L'ariavetro è il vero elemento di questa poesia, e la città mentale in cui la situo è una città di vetro, che si fa diafana fino a che scompare. È la determinatezza del medio che sbocca nel senso del nulla (mentre in Leopardi è l'indeterminatezza che raggiunge lo stesso effetto). O per esser più precisi, c’è un senso di sospensione ....(1032-33)

Il senso di sospensione, aggiunge Calvino, è dato dal "Forse" iniziale. Come il "Se" di Se una notte d'inverno. Anche qui qualcuno che va, qualcuno in cammino nell'aria gelida dell'inverno, verso qualcosa che resta sospeso per la durata di nove romanzi interrotti, fino al decimo in cui il "viaggiatore" approda ad un'ennesima città che sorge e svanisce nel ghiaccio, nell'irrealtà dell'aria di vetro, nella trasparenza del cristallo, degli elementi "determinati" e "diafani" di cui sono composte le calviniane "Città invisibili": i castelli della mente, le "metafore d'assenza". Come le città di Marcovaldo e di Montale anche quest'ultima si regge sul niente: "Salto da una sponda all'altra, e in basso non vedo alcun fondo ma solo il nulla che continua giù all'infinito; corro su pezzi di mondo sparpagliati nel vuoto; il mondo si sta sgretolando..." (Se una notte 254).

Il panico del narratore è il "terrore di ubriaco" di Montale ("il nulla alle mie spalle, il vuoto dietro / di me, con un terrore di ubriaco") che Calvino vede come l" "altra faccia" del "miracolo". Miracolo è la rivelazione del nulla contrapposta all" "inganno consueto" della presenza del mondo: "La scoperta è salutata dal poeta con favore ... ma anche sofferta come vertigine spaventosa ..." ("Forse un mattino" 1034). In "Quale storia laggiù attende la fine?", nell'ultima metamorfosi del "viaggiatore", c’è un Marcovaldo, per il quale la scomparsa del quotidiano è l'apertura dell'orizzonte del desiderio, e c'è un "ubriaco", che barcolla nella vertigine dell'impossibile: da una parte lo spazio che si stende tra la realtà e l'illusione, dall'altra la voragine tra la realtà e il nulla.

L'intero romanzo Se una notte d'inverno un viaggiatore si sostiene sull'accavallarsi di queste ipotesi. Tra la "quotidianità" della cornice e la "lettura" c'è lo spazio dell'illusione. ${ }^{4}$ La via di fuga del "Lettore" e della "Lettrice" è nella "pagina" in cui si iscrivono mondi possibili: la tensione è tra realtà e immaginario, città visibile e città invisibile, "villaggio" e "Castello". Ma nei romanzi inseriti nella comice il personaggio è colui che vive sull'abisso del nulla. Per lui il mondo è una facciata, lo "schermo"5 su cui si muovono figure provvisorie in bilico tra quello che è e quello che sta per essere: il "mistero", il punto di passaggio che ne stravolgerà il destino. L'"essere" è là, è quello che non appare, è quello a cui tutti i segni rimandano ma che non si può definire, afferrare.

Tutto ciò è esplicito nella narrazione di Calvino: questo suo personaggio lo sa, lo racconta dal momento in cui dice "Io", in cui si rivela come colui che vive nell'attesa tra passato e futuro, un viaggiatore "che ha perso una coincidenza" (16), che aspetta in una stazione, "questa specie di limbo illuminato sospeso tra le due oscurità del fascio dei binari e della città nebbiosa" (14). Una situazione esistenziale che mentre si dichiara normale ("esperienza di tutti"), afferma la 
propria "differenza"; la differenza del "rischio", 6 del "pericolo" (16) di una situazione limite, sospesa tra le due incognite del passato e del futuro che sono fuori dal romanzo; il rischio di identificarsi con chi dice: "Io appunto mi trovo qui senza un qui né un altrove" (17).

L'Io che si muove da un inizio di romanzo all'altro è al confine di una metamorfosi, in lotta con un alter-ego, si vive come "bifronte". La "vertigine", il "precipizio", la "breccia", lo "squarcio", sono ciò di cui esiste: è il protagonista del vuoto, l'equilibrista dei fili sospesi. In "Fuori dell'abitato di Malbork" è lui che richiama il lettore sul "senso della perdita, la vertigine della dissoluzione" (35). Il suo viaggio nel futuro inizia con una porta aperta su "una nuvola di vapore nell'aria di gelido vetro", quella che nel commento calviniano di Montale è la magica formula di passaggio al nulla. In "Sporgendosi dalla costa scoscesa" scrive di aver "provato un senso di vertigine, come non facessi che precipitare da un mondo all'altro e in ognuno arrivassi poco dopo che la fine del mondo era avvenuta" (55). Per lui la natura vera delle cose si rivela "solo nello sfacelo" (56); afferma di cercare "la via d'un'evasione, forse d'una metamorfosi, d'una resurrezione" (63); sente che nell'ordine perfetto dell'universo si è "aperta una breccia, uno squarcio irreparabile" (66). In "Senza temere il vento e la vertigine": "In un turbamento che dura un istante, mi pare di stare sentendo quel che lei sente: che ogni vuoto continua nel vuoto, ogni strapiombo anche minimo dà su un altro strapiombo, ogni voragine sbocca nell'abisso infinito" (82). In "Una rete di linee che s'allacciano" vorrebbe trasmettere "il senso d'essere indifeso, senza riparo in presenza di qualcosa che ci raggiunge da spazi estranei e sconosciuti" (133); mentre nell'inizio precedente, "Guarda in basso dove l'ombra s'addensa", avverte che la banalità di quella storia è una strategia narrativa per indicare indirettamente la foresta "che s'estende da tutte le parti e non lascia passare la luce tanto è folta" (109) del narrabile che resta al di là del romanzo: le "altre storie che potrei raccontare e forse racconterò o chissà che non abbia già raccontato in altra occasione ..." (109).

Insomma, raccontandosi e riraccontandosi, il viandante-narratore, lo "Scrittore"-personaggio (quel "lui" che diventa immediatamente "io"7 perché nonostante i "pericoli" e i "rischi", ed anzi a causa di essi, è in quell" "io" che vuole perdersi il "tu" del Lettore, il "lei" della Lettrice, l" "io" dell'autore vero e quello dell'autore implicito, come quello dell'universo dei dialoganti e degli interpretanti che ruotano dentro e intorno a ciò che nel testo si chiama "romanzo"), questo scrittore dunque, finisce per riscrivere sempre la stessa storia. Le "tracce" sono seminate ovunque per il lettore: un nome ripetuto da una storia all'altra, lo stesso vocabolario, la stessa situazione. ${ }^{8}$ Può cambiare lo scenario, l'ambiente, la circostanza, il dettaglio: perché "il romanzo" è in realtà "i romanzi"; ma questa voce narrante che si narra, da una stazione di provincia, da una cucina di campagna, da una città dell'Est, da un campus americano o dal Giappone ha una sola avventura da raccontare. La vicenda è personale, individuale ma insieme "specchio che riflette altri specchi", "caleidoscopio dei destini", perché, in 
quanto spazio del romanzo non è, come ci ricorda il protagonista, né il qui né l'altrove: è la zona bianca, è l'attesa tra "la città" e "il fascio dei binari". Il "romanzo" si pone qui come negazione della "contingenza"; l'altra faccia dell" incontro" e della "trasformazione" che si verifica nell'esperienza:

Come possiamo accettare, trasformandolo, il destino? Cosa è questa forza che ci permette di essere deboli, e questa nuova debolezza che sembra la forza più grande? ... Una zona dell'esperienza è avvistata da Nietzsche: lì non valgono più le misure abituali, le categorie normali. C'è un enigma da sciogliere: e non riguarda-come si potrebbe credere-un'esperienza eccezionale. Non c'è bisogno di salire in cima al monte o di scendere nel fondo dell'abisso. L'esperienza è la nostra, quella di ogni giorno. (Rovatti 33)

Il problema affrontato qui da Pier Aldo Rovatti è lo stesso che prende forma nel romanzo di Calvino: come coniugare la necessità e l'impossibilità, il "sempre" e il "mai", l'illusione e la realtà. È possibile un sapere di quella regione in cui l'ordine si incontra col disordine, il "globale" con il "locale", l'uguale con il diverso?: "È possibile un sapere della contingenza?" (39). È questa la domanda che Rovatti individua (dopo Nietzsche) in Peter Handke come in Michel Serres e in Jacques Lacan.

In Handke ciò che balza in primo piano è la "normalità" come insieme di "banalità", di azioni apparentemente insignificanti, legate tra loro da scarti casuali, da piccole "catastrofi" che spiazzano: "Piacere di ciò che è normale: difficile conquista di un tale piacere che pure sembra a portata di mano . . . Scoprire la 'normalità' è appunto la scoperta dello spezzettamento dell'esperienza, tessuto oscillante di piccole mosse che esige un'osservazione diversa . . .". C'è un percorso che è insieme "casuale" e "significativo"; c'è la "memoria", il "deposito dei vissuti", ma non intacca l'atomizzazione del quotidiano. Il viaggio nell'esperienza è fatto di "incontri" e di "accordi" mancati. Ma si tratta davvero di accordi mancati o dell'unico accordo possibile a partire dalle differenze?

Così per Serres perde di significato il viaggio lineare, razionale, verso una meta. Per il suo Zenone non c'è più Elea: ${ }^{9}$ ha capito che la ragione non è universale ma singolare, che "il 'globale' è un 'locale' storicamente sedimentato", che il viaggio non ha sbocchi, che "Elea (cioè la nostra identità) sta piuttosto nel viaggio stesso: in una randonnée, dice Serres, un girare, un giro, un andare in giro, ma anche random, caso, scelta, chance, e certamente rischio" (38). Ecco dunque che il bisogno di comprensione si sposta dall" "ordine" verso il "disordine", a quell'altro senso che non è riducibile a un modello. Rivolgere lo sguardo alla "vita" e alla "storia" vuol dire assumerne la complessità: ". . . contingenza e disordine non ne sono i residui irrazionali, ma le chiavi di accesso" (39). La metafora è sempre il viaggio: è una nuova "iniziazione" con diverse "prove". Se la domanda di Rovatti e di Serres diventa dunque: "E possibile un sapere della contingenza?" e, indirettamente: "Riusciremo a comprendere entro quale viaggio stiamo muovendoci?" (39), il romanzo di Calvino prospetta una questione simile: "È possibile una 'formazione' della contingenza?". 
Sul romanzo come "iniziazione" è Giuliano Gramigna che ci riporta le parole di Calvino: "Potremo costruire romanzi 'artificiali' . . potremo giocare al romanzo come si gioca a scacchi, con assoluta lealtà. Ma siccome gli schemi del romanzo sono quelli di un rito di iniziazione . . . il romanzo finirà per coinvolgerci nostro malgrado autore e lettore, finirà per rimettere in gioco tutto quello che abbiamo dentro e fuori ..." (5). E in quale direzione si collochi la ricerca di Se una notte d' inverno ci viene suggerito da Calvino stesso in quel capitolo 8 che costituisce (secondo le dichiarazioni dell'autore citate da Segre) il "nucleo generativo" del romanzo. La crisi di "senso" dello scrittore Silas Flannery si situa nell'impossibilità di coniugare due "programmi" opposti: da una parte c'è lo scrittore che dice "La sola verità che posso scrivere è quella dell'istante che vivo"; dall'altra, lo stesso scrittore "insoddisfatto" che afferma: "Ma io non credo che la totalità sia contenibile nel linguaggio; il mio problema è ciò che resta fuori, il non-scritto, il non-scrivibile" (181). È ancora, e sempre, la parabola di Calvino dalla "nausea" alla "vertigine", e se il primo programma, quello di "descrivere" l'istante si era con Palomar praticamente esaurito, ${ }^{10}$ la sfida di oggi diventa il romanzo del non-scrivibile che si fa contingenza; della contingenza come testimonianza del non-scrivibile. ${ }^{11}$

È con Lacan, ci ricorda Rovatti, che ci troviamo a "costruire" là dove le teorie si indeboliscono. Se non ci sono più ragioni forti questo non significa la perdita totale del senso. Si tratta di guardare all'esperienza: "Necessità e impossibilità, dice Lacan, possono, anzi devono coniugarsi: l'una e l'altra, nel loro tagliarsi, mutano identità. E il soggetto con loro" (40).

Per Lacan l'importante di ciò che è stato rivelato dal discorso psicanalitico è nel rapporto tra il sapere e l'amore. È nel rapporto d'amore che due "saperi inconsci" si incontrano: il sapere inconscio della "necessità" (della idealizzazione dell'amore nel "sempre") e dell'"impossibilita" che è invece del reale. Lacan definisce con la formula "non cessa di scriversi" il "necessario", che non è il reale. Il reale è caratterizzato dalla "impossibilità", cioè da una conoscenza, da un "godimento" sempre inadeguato rispetto al "sapere inconscio". La "contingenza", definita da Lacan come ciò che "cessa di non scriversi", è il risultato momentaneo di una conciliazione tra quei due opposti, reso possibile dal loro reciproco indebolimento. La contingenza sembra vista da Lacan come il prodotto di una "maturita" dell'inconscio in grado di "riconoscere" impossibilità e illusioni e di costruire su di ciò un "senso" che non si presenta né come rinuncia né come compromesso. Il suo "incontro" non sorge infatti sull'occultamento dell'impossibilità o sulla rinuncia all'illusione, ma conserva in sé la traccia di entrambi. Il necessario, il "sempre", viene assunto nella contingenza nell'unica forma possibile, come "sostituzione" che si opera in un momento di "sospensione". E in questo momento che "Ogni amore, non sussistendo che nel "cessa di non scriversi', tende a far passare la negazione al 'non cessa di scriversi', non cessa, non cesserà" (Lacan 146). Questa condizione viene descritta da Lacan in termini di "dramma" e di "destino". 
Necessità e impossibilità sono agli antipodi, come il "sempre" e il "mai". Eppure sono entrambe qualità dell'amore (come di ogni "esperienza"). L'incontro avviene nel "momento", nel "qui" e "ora" della contingenza, ma per attuarsi ha bisogno dell'illusione di eternità e, insieme, della consapevolezza del limite del reale. È un momento di "riconoscimento", come dice Lacan, di questi due estremi e di "coraggio" nell'accettazione di un destino. L'incontro si crea nel "sapere" che la fuga verso l'illusione assoluta del "sempre" porta all'idealizzazione dell'incontro mancato; è 1" "impossibilità" del reale che permette l'ancoraggio al presente, che crea la contingenza: “. . . qui non c'è altro che incontro, l'incontro nel partner dei sintomi, degli affetti, di tutto quel che in ciascuno segna la traccia del suo esilio ... qualcosa si incontra che può variare infinitamente quanto al livello del sapere, ma che, per un istante, dà l'illusione che qualcosa ... si iscriva nel destino di ognuno ... Lo spostamento ... dalla contingenza alla necessità, ecco il punto di sospensione cui ogni amore si attacca" (Lacan 145-46). Nell'inconscio si opera una sostituzione: ci si illude del "sempre" (ma nella consapevolezza della sua impossibilità) e si coglie 1'“ora".

Si chiede Rovatti che cosa mantenga la contingenza, che cosa permetta che le due tracce d'esilio "non esplodano in direzioni opposte, non diventino veri esili, estranei e lontani" (41). È appunto l'indebolimento del sempre, è la "moderazione" di cui parla Nietzsche; è il peso del reale che impedisce l'illusione del senza limite: "Se la realtà non si rivelasse impossibile, crederemmo nella necessità assoluta e onnipotente..." (Rovatti 41).

Così è per il protagonista di Calvino nell'ultimo dei romanzi interrotti di Se una notte d' inverno un viaggiatore. Si intitola "Quale storia laggiù attende la fine?" ed è una storia sull'incontro del sempre e del mai, del necessario (ciò che non ha fine) e dell'impossibile (ciò che deve finire). Il romanzo deve finire, la storia del Lettore e della Lettrice deve concludersi. La voce narrante (quell'aspetto della voce narrante che ha preso la forma di un personaggio-scrittore sospeso nel labirinto del proprio racconto) deve finire il proprio discorso.

Il discorso è una parabola che parla dell'illusione del potere, del controllo assoluto sull'esperienza, che si scontra con la realtà del limite. Da questo "riconoscimento" nasce l'"incontro", il rapporto con l'altro, con la quotidianità, con ciò che è "fuori" dal romanzo. Il romanzo ${ }^{12}$ diventa allora in Calvino il regno dell'inconscio che il Lettore e la Lettrice attraversano per riconoscere l'illusorietà della "via di fuga" dell'infinita possibilità e l'abisso del niente. È una "formazione" che li porta a quello che apparentemente è il finale tradizionale del Bildungsroman classico.

Secondo Franco Moretti: “. . . nel 'romanzo familiare' della tradizione inglese, e nella forma classica del Bildungsroman-le trasformazioni narrative trovano il loro senso nel condurre ad un finale particolarmente marcato ... Questa retorica teleologica-a dare senso agli eventi è sempre e soltanto il loro scopoè l'equivalente normativo del pensiero hegeliano, cui l'accomuna d'altronde una spiccata vocazione normativa: gli eventi acquistano senso nel condurre a uno 
scopo, e uno soltanto" (16). È un romanzo che Moretti assimila alla "favola" nella sua ricerca delle certezze contrapposte ai mutamenti e sappiamo come la favola sia l'eterno modello invocato a proposito della narrativa di Calvino. Questo è particolarmente vero nel caso di Se una notte d' inverno e soprattutto in riferimento al matrimonio finale, direttamente identificato dai più con il tradizionale "e vissero felici e contenti". ${ }^{13} \mathrm{Ma}$ c'è una differenza: in Se una notte, il matrimonio, l"“incontro", l'“accordo" è il risultato di un "attraversamento"; dell'identificazione con il protagonista dei "romanzi"; dell'accettazione del "rischio" di cui il personaggio parla nel presentarsi, nel riconoscersi come il portatore di una dimensione in bilico sul nulla, di un "rotolare fuori centro":

... che altro è la perdita del centro se non la dichiarazione, la sanzione che il pensiero "forte" è ormai insostenibile? La situazione tipica del pensiero "forte" è infatti quella in cui pensante e pensato, chi pensa e cosa si pensa sono solidali: si tengono in una stretta, in una corrispondenza speculare. La situazione che Nietzsche vede è caratterizzata, invece, dalla possibilità di perdersi: l'uomo è giunto dinanzi a un limite, un passo oltre e potrà sprofondare, perdersi completamente. Il luogo in cui il senso potrà riattivarsi è avvistabile solo di qui, drammaticamente. (Rovatti 29-30)

Per Rovatti l'alternativa è tra la rinuncia (dichiarare invalicabile questo limite) e l'avventurarsi in un "cammino" difficile. Il personaggio di Calvino è "in cammino", un cammino di cui l'ultima storia sembra indicare il "senso", la possibile direzione.

Anche in questa storia c'eे un incontro, quello che in tutti i nove precedenti romanzi interrotti è sullo sfondo: un incontro potenziale, ma in realtà impossibile; un incontro mancato: due "tracce d'esilio" che si sfiorano, si mancano, esplodono in direzioni opposte. Perché il personaggio vive tra la fuga del sempre e l'abisso del mai: quel rapporto è il "tutto" verso cui corre il romanzo e il niente su cui invariabilmente si sospende. Ė questo il "vero" viaggio? Quello che il Lettore e la Lettrice vanno cercando investendo la "quotidianità" nella ricerca del "romanzo"? Ma la ricerca del romanzo è in realtà la ricerca del suo finale. C'è bisogno di "finale", c'è bisogno di "incontro".

Così il protagonista di "Quale storia laggiù attende la fine" nel suo cancellare la realtà minuta, limitata, soffocante, nel suo tentativo di cancellare il "limite" e di tracciare possibilità infinite vuole salvare qualcosa del suo mondo: la "possibilità" dell'incontro, una possibilità che nelle sue intenzioni deve restare tale, casuale, non definita; reale ma senza i limiti della realtà.

... né io né lei facciamo mai nulla per provocare un incontro; forse perché sappiamo che non sarebbe più la stessa cosa. Ora, in un mondo semplificato e ridotto, in cui sia sgombrato il campo da tutte quelle situazioni prestabilite per cui il fatto che io e Franziska ci si veda più spesso implicherebbe una relazione tra noi che andrebbe in qualche modo definita, magari in vista di un matrimonio . . . una volta spariti tutti questi condizionamenti ... l'incontrare Franziska dovrebb'essere ancora più bello e piacevole... (248) 
Ma il "nulla" ha preso il sopravvento sul "tutto", la possibilità illimitata si è trasformata nel suo contrario, l'incontro svanisce con la scomparsa del suo contesto reale: “. . . la presenza del mondo amico e ostile, le cose di cui rallegrarsi o contro cui battersi .... Lo penso con tutte le mie forze ma ormai so che esse non bastano a farlo esistere: il nulla è più forte e ha occupato tutta la terra" (253). Quella che nella formula lacaniana è il "non cessa di scriversi", la creatività assoluta che per un momento ha illuso il protagonista, è ora il "non cessa di non scriversi": il vuoto, l'assenza.

Ma è proprio qui che Calvino, con un gesto in extremis, ci restituisce la contingenza. C'è un salto nel vuoto, nello spazio che si spalanca tra l'uomo e il suo "incontro", su quel mondo ridotto a "una crosta gelata", a un "foglio di carta dove non si riescono a scrivere altro che parole astratte..." (254). Ė quel balzo che decide, che afferra, che crea una nuova "scrittura". Non la scrittura eterna, ma quella del momento, che nel momento si illude di essere il "sempre" pur sapendo di rotolare verso il "mai".

Nella prima parte di Se una notte e soprattutto nel capitolo settimo, Calvino stabilisce un parallelo tra lettura e rapporto sessuale su cui molti hanno commentato. Così Wiley Feinstein: "Calvino's doubt is that reading, like sex, does not allow escape from separateness for either reader or author . . . Reader-author relationships are therefore just as doomed to failure as sexual relationships" (152). Non c'è dubbio che qui Calvino sia sulla stessa posizione di Lacan il quale afferma: “Non c'è rapporto sessuale perché il godimento dell'Altro preso come corpo è sempre inadeguato-perverso da un lato, in quanto l'Altro si riduce all'oggetto ' $a$ ' - - dall'altro, dirò folle, enigmatico" (Lacan 145); ma Lacan (e così Calvino) non si ferma qui: "Non è forse dallo scontro con questa impasse, con questa impossibilità da cui si definisce un reale, che è messo alla prova l'amore? Del partner, l'amore può realizzare solo ciò che ho chiamato con una sorta di poesia, per farmi intendere, il coraggio, nei confronti di questo destino fatale" (145).

"Labor of love", ci ricorda Teresa de Lauretis, è la definizione di Calvino del "lavoro della scrittura" (82) ed è qui che in Se una notte si stabilisce il rapporto tra la vicenda dei protagonisti e l' "incontro" autore-lettore. Non è un caso che il rapporto sessuale non coincida con il finale del libro (è infatti a metà del testo), ma stabilisca invece il parallelo tra due ricerche che continuano: per la Lettrice quella del "partner fantasmatico", dell"“apocrifo visitatore dei suoi sogni" (Se una notte 156); per lo "scrittore" quella della sua "vocazione": "Forse la mia vocazione vera era quella d'autore d'apocrifi, nei vari significati del termine: perché scrivere è sempre nascondere qualcosa perché venga poi scoperto; perché la verità che può uscire dalla mia penna è come una scheggia saltata via da un grande macigno ...." (193). Dal "piacere del testo" al testo come conquista difficile di frammenti di verità. Il parallelo testualità-sessualità è dunque per Calvino non tanto, come Feinstein ed altri hanno osservato, un pretesto per umorismo, ma un invito ad andare oltre. D'altra parte anche Segre metteva in guardia contro 
un'interpretazione statica: "Tuttavia la forte emergenza dell'eros, così come le riflessioni sul manqué, la presenza non dichiarata ma evidente del concetto di pulsione, ci insinuano che sarebbe parziale, e in sostanza falsa, un'interpretazione statica... Il libro è invece percorso da turbamenti. Anche da vertigini e oscuri presentimenti" (209).

Il Lettore e la Lettrice imparano la lezione e si sposano. Troppo banale per Calvino, come qualcuno ha osservato, se si trattasse comunque di un "lieto fine". Ma non è così. L'ultimo quadro della cornice ci presenta la nuova quotidianità del Lettore e della Lettrice: "Un grande letto matrimoniale accoglie le vostre letture parallele" (262). Ma Ludmilla, l'infaticabile Lettrice, è stanca di leggere e spegne la sua luce. Il Lettore continua solitario: sta per finire Se una notte d'inverno un viaggiatore. È una sceneggiatura sommessa, non lontana dal malessere degli Amori difficili. ${ }^{14}$ Nessun trionfalismo, nessuna favola di armonia raggiunta: "L'accordo è comunque difficile, mai raggiunto completamente, richiede un viaggio complicato" (Rovatti 36). Il viaggio, metaforizzato da Calvino nella lettura, continua. Il "finale", l'incontro, è sempre provvisorio, si riapre su orizzonti di avventure in mondi possibili, l'altra faccia della quotidianità, l'unica che la renda vivibile. La lettura è qui un percorso iniziatico, è il viaggio nell'inconscio, negli inferni, nelle paure degli abissi. O nei sogni, nei miti, nelle illusioni. È il luogo in cui rinnoviamo la conoscenza del nostro essere in bilico.

La situazione rappresentata da Calvino nei dieci inizi di romanzo è la stessa descritta da Rovatti in riferimento a Nietzsche: “. . . l'immagine è quella di una situazione di equilibrio instabile su una piccola superficie d'appoggio, uno stare in bilico che può produrre ugualmente lo sviluppo della massima potenzialità e i] definitivo sprofondamento. Non è più una dialettica, ma un cammino che si biforca senza soluzione. Le due possibilità stanno insieme: questo bilico è, secondo Nietzsche, la situazione dell'uomo contemporaneo. Come può una simile precarietà essere la massima forza?" (31-32). La forza si identifica con la debolezza: bisogna aggrapparsi ad un appiglio per non cadere, ma è questo appiglio che permette di coniugare nell'attimo di contingenza la pesantezza e la leggerezza, di attraversare la "vertigine" per conoscerla.

È il "matrimonio" finale una resa alla realtà e alle sue convenzioni o, come l" "incontro" di Lacan, un esempio di quella traccia di conoscenza che permette l'esistenza del reale, di quei nuclei di esperienza che si creano tra l'intuizione di un desiderio e la percezione del suo limite?

Questo "sapere della contingenza" è, per Lacan, "il destino e il dramma dell'amore" (146). Per Calvino è la possibilità di un diverso "finale"; di una diversa "formazione".

University of California at Berkeley

\section{NOTE}

1 Per quanto riguarda il personaggio della Lettrice cfr. anche Ferretti il quale vede in Lud- 
milla "il personaggio centrale di tutta l'operazione" (130) intesa come adulazione del lettore medio disinteressato, ma anche aproblematico, per una precisa scelta di mercato. Non condivido questo giudizio che non mi sembra motivato né dal testo né dalle dichiarazioni di Calvino (riportate da Ferretti). Direi invece che la scelta di "medietà" della Lettrice (e del Lettore) va nella direzione di quella "normalita" che vuole essere la caratteristica dei due protagonisti della comice contrapposta al "romanzesco" dei personaggi dei romanzi inseriti. Inoltre Calvino gioca con le immagini dei media (la sua Ludmilla è la donna rappresentata e presupposta dalla pubblicità e dalle riviste femminili: cfr. descrizione scopertamente leziosa 143-44), e con gli stereotipi (vedi la sorella-antitesi Lotaria). Insomma le "sublimazioni" di Calvino sono sempre relative e se c'è una "musa" non è la Lettrice ma la "lettura". Per un discorso più generale sul ruolo della Musa ispiratrice nella letteratura, vedi Maria Corti 9-11.

2 Scrive Luisa Guy che la cornice "... ironically negates the idea of openness suggested by the ten unfinished novels" (66). Secondo questa interpretazione la storia del Lettore e della Lettrice ha un finale tradizionale dato dal matrimonio e dalla conquista del tesoro perduto, in questo caso il romanzo di Calvino che il Lettore si propone di finire nell'ultima pagina di Se una notte. Ma la "conclusione" di Calvino mi sembra molto più ambigua (non abbiamo nessuna garanzia che il libro tra le mani del Lettore questa volta sia davvero il romanzo di Calvino né tanto meno che riesca, come noi, a finirlo: la storia più che finire ricomincia), e il matrimonio finale, come anticipa a metà del romanzo la voce narrante, è solo la promessa di una "debole" e provvisoria armonia: "Domani, Lettore e Lettrice, se sarete insieme, se vi coricherete nello stesso letto come una coppia assestata, ognuno accenderà la lampada al suo capezzale e sprofonderà nel suo libro; due letture parallele accompagneranno l'approssimarsi del sonno; prima tu poi tu spegnerete la luce; reduci da universi separati, vi ritrovate fugacemente nel buio dove tutte le lontananze si cancellano, prima che sogni divergenti vi trascinino ancora tu da una parte e tu dall'altra ..." (157).

3 Vedi Paolo Fabbri: “. . . nausea e vertigine: tra questi patemi si gioca la posta della sua ricerca e del suo stile ..." (28). È una ricerca che colloca Calvino all'interno di quel discorso secondo cui: "L'essere il cui senso si tratta di recuperare è un essere che tende a identificarsi con il nulla, con i caratteri effimeri dell'esistere, come rinchiuso fra i termini della nascita e della morte" (Vattimo, La fine 129).

4 Vattimo: "In qualche modo la verità possibile dell'arte consiste nel presentare un'alternativa al mondo reale, ed in questo senso la chiamo illusione" (Filosofia 59). Vattimo riprende da Heidegger l'idea dell'esperienza estetica come "dislocazione", come "rappresentazione di una molteplicità di possibilità di esistenza che rendano meno cogente la nostra singola esistenza" (64); il che concorda con quanto dichiarato da Calvino in una delle sue ultime interviste. A Nascimbeni che gli poneva la stessa domanda rivolta a Borges anni prima ("Perché tenta di figurarsi altre vite diverse dalla sua? Perché anche lei, in un certo senso, vorrebbe essere stato "con i bisonti dell'aurora'?"), rispose di riconoscersi nella risposta di Borges (che affermava di inseguire quelle fantasie perché era un po' stanco di essere Borges) aggiungendo: "Sono sempre insoddisfatto. Cerco di immaginare dove mi avrebbe portato l'altra strada, quella che non ho scelto. Così, anziché pensare ai libri che sarebbe naturale scrivessi, mi capita di pensare ai libri che non so e non posso scrivere, ai libri che scriverebbe un altro ... Ma c'è anche il desiderio, la necessità di interpretare la nostra epoca in maniera più rivelatrice..." (3).

5 L'immagine è di Montale e Calvino così commenta: "L'illusione del mondo veniva tradizionalmente resa da poeti e drammaturghi con metafore teatrali; il nostro secolo sostituisce al mondo come teatro il mondo come cinematografo, vorticare d'immagini su una tela bianca" ("Forse un mattino" 1037). Per Calvino la metafora, da Marcovaldo a Se una notte, diventa la "pagina bianca": il mondo come romanzo e il romanzo come "mondo".

6 L'uso da parte di Calvino del termine "rischio" per designare la condizione del perso- 
naggio-io e la sua funzione di mediazione tra il lettore (e l'autore) e ciò che resta inconoscibile (". . . ed è questo qualcosa d'altro che rende rischioso identificarsi con me, per te lettore e per lui autore...", Se una notte 16), non sembra casuale. Cfr. Vattimo, Al di là del soggetto: ". . . Heidegger analizza anche la condizione del poeta in termini di rischio, perché la sua funzione è quella di mettere il mondo dell'esperienza articolata, delle mediazioni, in relazione al Caos originario ... il poeta, incontrando l'essere come physis e temporalità vissuta, incontra il proprio essere-per-la-morte, l'alterità radicale che gli si dà come il nulla e il silenzio" (90).

7 Cfr. Calvino, "ll romanziere e il suo suggeritore": "La cultura moderna ha elaborato questa categoria molto ingombrante e scomoda che è l'io. Lo scrittore, sapendo di avere un io, si pone il problema: o di scrivere con l'io, convincendosi che l'io sia uno strumento della scrittura; oppure di non sapere dove metterlo, questo io, durante la scrittura" (5). In Se una notte, questo io identico e diverso uno e molteplice diventa la traduzione del programma annunciato da Silas Flannery, il personaggio-scrittore che nel romanzo prende la parola in quanto scrittore: "Anch'io vorrei cancellare me stesso e trovare per ogni libro un altro io, un'altra voce, un altro nome, rinascere; ma il mio scopo è di catturare nel libro il mondo illeggibile, senza centro, senza io" (180). Il che non si discosta dalle conclusioni dello stesso Calvino nell'articolo citato: “. . . lo scrittore al momento in cui mette mano a un'opera indossa una maschera, assume una voce, entra in un personaggio che è un se stesso speciale...".

8 Per ulteriori esemplificazioni dei rimandi da una storia all'altra vedi Nuccia Bencivenga (7-9). Vedi inoltre Segre: "Per questo è facile rilevare come i dieci romanzi inseriti ... riescano a rappresentare una compatta unità di esplorazione"(208).

9 Da notare che Zenone di Elea compare anche nel testo di Calvino, all'inizio della vicenda del viaggiatore, come parola d'ordine che avrebbe permesso il riconoscimento dell'altro e la felice conclusione del viaggio. Ma la parola non sarà mai pronunciata e il viaggiatore, come lo Zenone di Serres, rimane senza meta, nel "caso" e nel "rischio".

10 A proposito di Palomar come progetto precedente a Se una notte d'inverno un viaggiatore vedi il mio recente articolo "Palomar e il viaggio di Calvino dall" "esattezza' alla 'leggerezza'” (Italica, Winter 1992).

11 Per una discussione sulla visione della scrittura in Calvino-Flannery "al limite del sacro" vedi il saggio di Olds.

12 Il romanzo che il Lettore e la Lettrice leggono in quanto personaggi, ma anche Se una notte d'inverno, come romanzo letto dal lettore reale che, pur attraverso la mediazione e distanza dei "lettori" e degli "autori" fittizi, percorre il proprio viaggio nel "romanzo".

13 Cfr. tra gli altri JoAnn Cannon: "In this most traditional of possible endings, boy and girl marry and live happily ever after" (108). Per la Cannon questa conclusione esprime il desiderio di Calvino di incorporare la parte nel tutto, di controllare il dialogo tra il testo e il lettore, di diventare portatore di significato: "But this strategy is unsuccessful; rather than bringing the text back within the author's sphere, it merely underlines the degree to which the text cannot be controlled or closed by the author" (108). Ma non si capisce perché Calvino avrebbe scelto una strategia così perdente per dimostrare il contrario di quanto la sua produzione critica e letteraria andava da tempo affermando. Mi sembra invece che Calvino di proposito usi la più banale e scontata delle chiusure proprio per sottolinearne la valenza completamente diversa nel contesto di questo romanzo e di quella condizione postmoderna a cui continuamente si richiama. Se il romanzo si conclude, se l" "incontro" avviene è proprio perché avendo perso le caratteristiche definitive e definitorie (ed è questa la strategia di Calvino nei romanzi iscritti come nella comice), può essere di nuovo desiderato, come equilibrio momentaneo, soluzione provvisoria, esperienza contingente destinata ad aprirsi su altre molteplici esperienze. Per una interpretazione della "favola" di Calvino orientata nel senso del "caos" e della "molteplicità" vedi il seggio di Beard.

14 Il riferimento agli Amori difficili è di Teresa de Lauretis nel saggio citato in cui discute $\mathrm{Se}$ 
una notte d'inverno un viaggiatore come rifacimento postmoderno di uno dei racconti di quella raccolta: "L'avventura di un lettore". La questione centrale qui affrontata dalla de Lauretis è quella della rappresentazione della donna: "... the female reader here is finally re-contained within the frame of the book as merely a character in man's fiction, reduced to a portrait, an image, a figure of the male imaginary ..." (82). Eे un discorso molto importante e complesso che merita una trattazione a parte, e in riferimento a tutta la produzione di Calvino. Mi sembra infatti che tutti i personaggi femminili di Calvino, dalla Viola del Barone rampante, alle donne delle Cosmicomiche e delle Città invisibili come di Sotto il sole giaguaro, quei personaggi "positivi", "femminili" e "intelligenti", che, come la Ludmilla di Se una notte, hanno mandato in visibilio la maggior parte dei critici-uomini, abbiano, chi più chi meno, gli attributi stereotipi della femminilità che possiamo riassumere in "istintività", "forza naturale" contrapposta al raziocinio maschile. Questo è particolarmente evidente in Se una notte perché Ludmilla viene affiancata dalla sorella Lotaria, "The Non-Feminine Woman", la critica e scrittrice femminista dalla voce roca: "... a feature that in Italian literature since Pavese ... has come to denote symbolic castration" (76). Mi sembra però che proprio l'insistenza di Calvino sugli stereotipi potrebbe essere il modo tipicamente postmoderno di "prendere le distanze", riscrivendo la tradizione letteraria e l'immaginario dei media e spingendo il lettore a decostruime i feticci: a cercare le "altre" donne oltre la perfezione da copertina di Ludmilla e l'aggressività di Lotaria; a cercare gli "altri" finali oltre il "lieto fine" di Se una notte d'inverno un viaggiatore. Che è poi il risultato a cui arriva Teresa de Lauretis nella sua decostruzione del personaggio di Lotaria.

\section{OPERE CITATE}

Beard, P.W. "Once upon a Time in Calvino's If on a Winter's Night a Traveler". Italian Quarterly 115-16 (Winter-Spring 1989): 55-63.

Bencivenga, Nuccia. "Caliph, Travelers, and Other Stories". Forum Italicum 1 (Spring 1986): 7-9.

Calvino, Italo. "Forse un mattino andando". Il materiale el' immaginario 8. Eds. Remo Ceserani e

Lidia De Federicis. Torino: Loescher, 1983. 1032-37.

"Il romanziere e il suo suggeritore". Corriere della Sera (19 ottobre 1985): 5.

Marcovaldo ovvero Le stagioni in città. Torino: Einaudi, 1969.

Se una notte d' inverno un viaggiatore. Torino: Einaudi, 1979.

Cannon, JoAnn. Italo Calvino Writer and Critic. Ravenna: Longo Editore, 1981.

Corti, Maria. Il viaggio testuale. Torino: Einaudi, 1978.

De Lauretis, Teresa. "Calvino and the Amazons. Reading the (Post) Modem Text". Technologies of Gender. Bloomington and Indianapolis: Indiana UP, 1987. 70-83.

Fabbri, Paolo. "Le trame del Bagatto: arcani narrativi e orditi del dire". Narratori dell' invisibile. Simposio in memoria di Italo Calvino. Modena: Mucchi Editore, 1987. 23-33.

Feinstein, Wiley. "The Doctrinal Core of If on a Winter's Night a Traveler". Calvino Revisited. Ed. Franco Ricci. Ottawa: Dovehouse Editions, 1989. 147-55.

Ferretti, Gian Carlo. Le capre di Bikini. Calvino giornalista e saggista 1945-1985. Roma: Editori Riuniti, 1989.

Gramigna, Giuliano. "Il grande gioco creativo di farsi critico di se stesso". Corriere della Sera (19 ottobre 1985): 5 .

Guy, Luisa. "'Quale storia laggiù attende la fine?' The Lettore's Successful Quest in Calvino's Se una notte d' inverno un viaggiatore". Italian Quarterly 115-16 (Winter-Spring 1989): 65-71.

Lacan, Jacques. Il seminario. Libro XX. Ancora 1972-1973. Trad. Sergio Benvenuto e Mariella Contri. Torino: Einaudi, 1983.

Montale, Eugenio. "Forse un mattino andando in un'aria di vetro". Ossi di seppia. Milano: Mondadori, 1963. 60.

Moretti, Franco. Il romanzo di formazione. Milano: Garzanti, 1986. 
Nascimbeni, Giulio. "Sono un po' stanco di essere Calvino". Corriere della Sera (5 dicembre 1984): 3 .

Olds, Marshall. "Another Book, Another Author: Calvino, Flaubert, Mallarme”. The Review of Contemporary Fiction 6 (Spring 1986): 117-23.

Rovatti, Pier Aldo. "Trasformazioni nel corso dell'esperienza". Il pensiero debole. Eds. Gianni Vattimo e Pier Aldo Rovatti. Milano: Feltrinelli, 1983. 29-51.

Segre, Cesare. "Se una notte d'inverno uno scrittore sognasse un aleph di dieci colori". Strumenti critici 2-3 (ottobre 1979): 177-215.

Vattimo, Gianni. Al dì là del soggetto. Milano: Feltrinelli, 1990.

. Filosofia al presente. Milano: Garzanti, 1990.

. La fine della modernitd. Milano: Garzanti, 1985. 